\title{
Targeting angiotensinogen with RNA-based therapeutics
}

\author{
Liwei Ren ${ }^{\mathrm{a}, \mathrm{b}}$, Katrina M. Mirabito Colafella ${ }^{\mathrm{c}}$, Dominique M. Bovée ${ }^{\mathrm{a}}$, \\ Estrellita Uijl', and A.H. Jan Danser ${ }^{\mathrm{a}}$
}

\begin{abstract}
Purpose of review
To summarize all available data on targeting angiotensinogen with RNA-based therapeutics as a new tool to combat cardiovascular diseases.

\section{Recent findings}

Liver-targeted, stable antisense oligonucleotides and small interfering RNA targeting angiotensinogen are now available, and may allow treatment with at most a few injections per year, thereby improving adherence. Promising results have been obtained in hypertensive animal models, as well as in rodent models of atherosclerosis, polycystic kidney disease and pulmonary fibrosis. The next step will be to evaluate the optimal degree of suppression, synergy with existing renin-angiotensin-aldosterone system blockers, and to determine harmful effects of suppressing angiotensinogen in the context of common comorbidities, such as heart failure and chronic kidney disease.

\section{Summary \\ Targeting angiotensinogen with RNA-based therapeutics is a promising new tool to treat hypertension and diseases beyond. Their long-lasting effects are particularly exciting, and if translated to a clinical application of at most a few administrations per year, may help to eliminate nonadherence.}

\section{Keywords}

angiotensin, angiotensinogen, antisense oligonucleotides, drug adherence, renin, RNA interference

\section{INTRODUCTION}

Although more than 100 commercial drugs and drug combinations are available for the treatment of hypertension, a substantial proportion of the hypertensive population remains uncontrolled or suboptimally controlled. This could relate to nonadherence and/or drug ineffectiveness. The latter might be because of counterregulatory mechanisms (like a rise in renin [1]) that eliminate or diminish the initial blood pressure-lowering effect. Hence, given the deleterious consequences of uncontrolled blood pressure, there still is a need for novel treatment options that preferably are not counterbalanced by the upregulation of contractile mechanisms and circumvent nonadherence. An attractive option is targeting angiotensinogen (AGT) with RNA-based therapeutics. As all angiotensins stem from AGT, deleting AGT will suppress angiotensin (Ang) formation, even when renin levels rise. Furthermore, the dosing frequency with this approach might go down to a few times per year, thereby potentially reducing the clinical and economic burden of nonadherence.

\section{RNA THERAPEUTIC APPROACHES}

RNA-based therapies bind to RNA and change the expression of any protein, even those not amenable to traditional approaches involving small-molecule drugs. A number of RNA therapeutic approaches have been developed $\left[2^{-"}, 3^{-*}\right]$, with antisense oligonucleotides (ASO) that inhibit RNA translation and oligonucleotides that function via the RNA interference (RNAi) pathway being the most clinically relevant. ASO-based therapies utilize Watson-Crick's

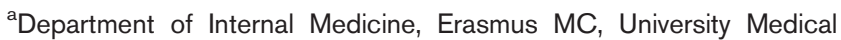
Center, Rotterdam, The Netherlands, ${ }^{\mathrm{b}}$ AstraZeneca-Shenzhen University Joint Institute of Nephrology, Department of Physiology, Shenzhen University Health Science Center, Shenzhen University, Shenzhen, China and ${ }^{\mathrm{C} C a r d i o v a s c u l a r}$ Disease Program, Biomedicine Discovery Institute and Department of Physiology, Monash University, Melbourne, Australia Correspondence to A.H. Jan Danser, PhD, Division of Pharmacology and Vascular Medicine, Department of Internal Medicine, Room EE1418b, Erasmus MC, Wytemaweg 80, 3015 CN Rotterdam, The Netherlands. Tel: +31 10 7043540; fax: +31 10 7044733;

e-mail: a.danser@erasmusmc.nl
}

Curr Opin Nephrol Hypertens 2020, 29:180-189

DOI:10.1097/MNH.0000000000000586 


\section{KEY POINTS}

- Liver-targeted, stable antisense oligonucleotides and small interfering RNA-targeting angiotensinogen, capable of suppressing angiotensinogen in a dosedependent manner, are now available.

- Suppressing angiotensinogen exerts similar effects as classical blockers of the renin-angiotensin-aldosterone system (RAAS) in hypertensive animal models, as well as in rodent models for atherosclerosis, polycystic kidney disease and pulmonary fibrosis.

- Future studies should evaluate their safety, for example, in the context of common comorbidities, such as heart failure and chronic kidney disease, and synergy with existing RAAS blockers.

- The long-lasting effects of this approach are particularly exciting, and if translated to a clinical application of at most a few administrations per year, may help to eliminate nonadherence. base-pairing rules and single-stranded DNA containing 15-30 nucleotides, which are designed in antisense orientation to the premRNA and mRNA of interest. Mature mRNA is formed in the nucleus by splicing the introns of premRNA. ASOs are able to modulate alternative splicing by binding to the premRNA producing different protein variants and changing the prevalence of one variant. When ASOs bind to their target mRNA, RNase $\mathrm{H} 1$ cleaves the RNA in an RNA-DNA duplex in both the cytoplasm and nucleus [4] destroying the mRNA and inhibiting the translation of the target protein (Fig. 1a). Chemically modified ASOs with phosphorothioate linkages can trigger RNA cleavage even in the absence of transfection reagent $[5,6]$. In contrast to the ASO approach, RNAi-based therapies utilize doublestranded RNA and exploit the RNAi process, an evolutionary conserved mechanism for the regulation of gene expression. At present, the majority of RNAi-based therapies employ noncoding small interfering RNA (siRNA) that are 21-23 bases in length and work naturally in the cell as a part of

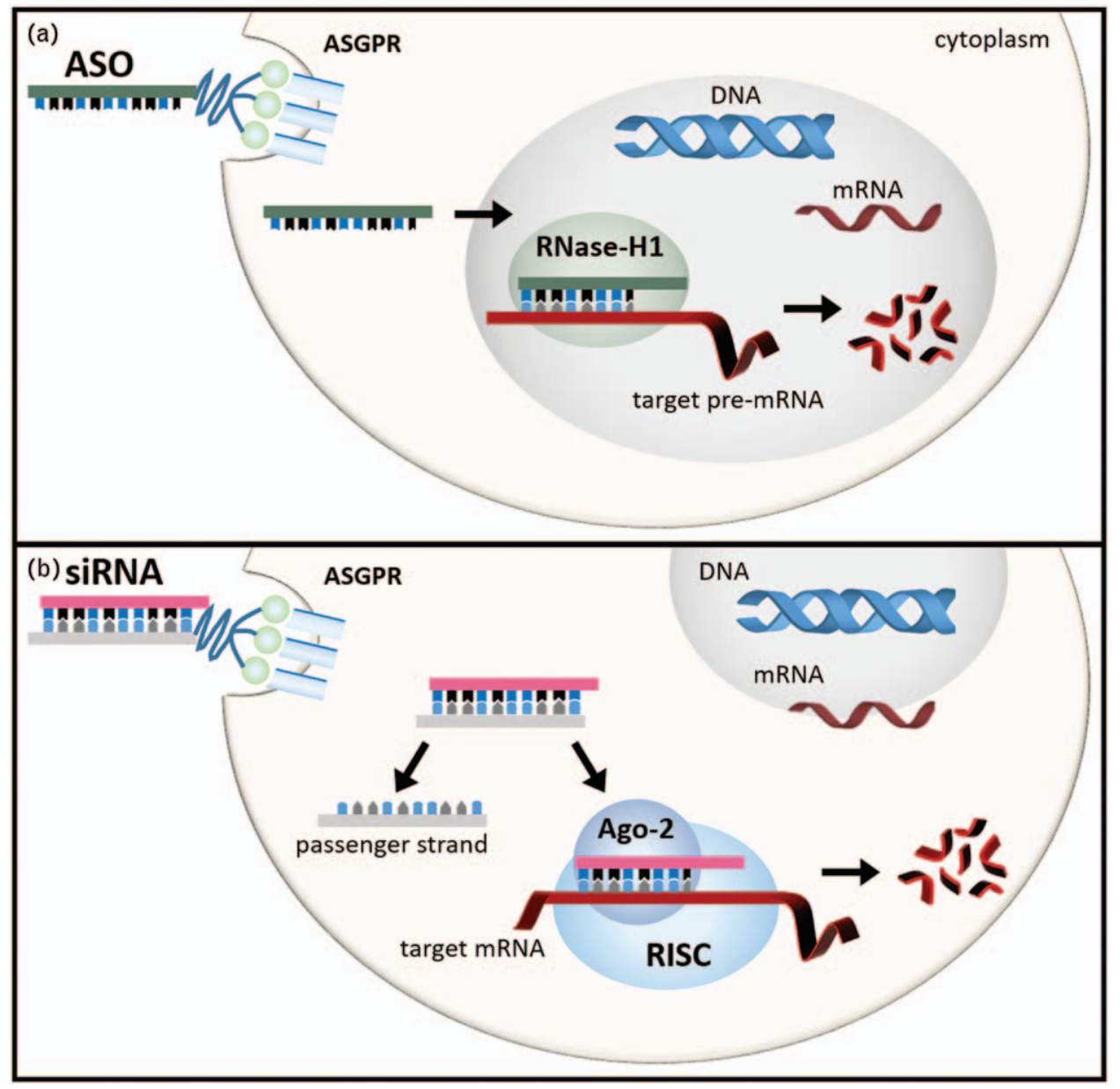

FIGURE 1. Inhibition of RNA translation by (a) antisense oligonucleotide or (b) RNA interference. See text for further explanation. Ago-2, argonaute-2; ASGPR, asialoglycoprotein receptor; RISC, RNA-Induced Silencing Complex. 
the RNA-Induced Silencing Complex (RISC). Unlike ASOs, when siRNA enters the cell it stays inactive until loaded by transactivation responsive RNA-binding protein into Argonaute where the passenger strand is removed and the remaining antisense strand binds complementary mRNA targets, leaving Argonaute endoribonuclease to cleave the mRNA, thus preventing protein translation [7] (Fig. 1b). In mammals, Argonaute contains an RNase $\mathrm{H}$ domain, but cleaves RNA in an RNA-RNA duplex [8]. The majority of RNA-based therapies in clinical pipeline utilize the RNAi approach (Table 1). This is unsurprising given that a major advantage of RNAi over ASO for therapeutic applications is that RNAi is much more potent and has a longer inhibitory effect [9].

The clinical translation of RNAi-based therapies has faced a number of challenges including off-target effects, siRNA delivery, immune reactions and toxicity [2"]. The biggest hurdle has been delivery as siRNAs do not readily cross the cell membrane. Various approaches have been proposed to solve the problem of siRNA delivery in vivo, for example, viruses, cationic lipids, polymers, and nanoparticles $[10,11]$. One of the most promising approaches to

Table 1. RNA-based therapies currently in clinical development

\begin{tabular}{|c|c|c|c|c|}
\hline Disease target & $\begin{array}{l}\text { Pharmaceutical } \\
\text { company }\end{array}$ & $\begin{array}{l}\text { Drug (alternate } \\
\text { name) }\end{array}$ & $\begin{array}{l}\text { Therapeutic } \\
\text { approach }\end{array}$ & $\begin{array}{l}\text { Stage of } \\
\text { development }\end{array}$ \\
\hline \multicolumn{5}{|l|}{ Cancer } \\
\hline \multicolumn{5}{|l|}{ Blood cancers } \\
\hline miR-155 & miRagen & $\begin{array}{l}\text { Cobomarsen } \\
\text { (MRG-106) }\end{array}$ & $\begin{array}{l}\text { RNAi } \\
\quad \text { (miRNA) }\end{array}$ & Phase $1 / 2$ \\
\hline \multicolumn{5}{|c|}{ Clear cell renal cell carcinoma } \\
\hline HIF- $2 \alpha$ & Arrowhead & ARO-HIF2 & RNAi (siRNA) & Pre-IND \\
\hline \multicolumn{5}{|l|}{ Cholangiocarcinoma } \\
\hline TGF- $\beta 1$ and COX2 & Sirnaomics & STP705L & RNAi (siRNA) & Phase 1 \\
\hline \multicolumn{5}{|c|}{ Nonmelanoma skin cancer } \\
\hline TGF- $\beta 1$ and COX2 & Sirnaomics & STP705 & RNAi (siRNA) & Phase 2 \\
\hline \multicolumn{5}{|l|}{ Pancreatic cancer } \\
\hline KRAS G12D & Silenseed & KRAS-LODER & RNAi (siRNA) & Phase 2 [23] \\
\hline \multicolumn{5}{|l|}{ Prostate cancer } \\
\hline Androgen receptors & lonis & $\begin{array}{l}\text { ARRx (IONIS-AR- } \\
2.5 \mathrm{Rx} \text { ) }\end{array}$ & ASO & Phase $1 / 2$ \\
\hline \multicolumn{5}{|l|}{ Various cancers } \\
\hline STAT3 & lonis-AstraZeneca & $\begin{array}{l}\text { Danvatirsen } \\
\text { (ISIS481464) }\end{array}$ & ASO & Phase $1 / 2$ \\
\hline \multicolumn{5}{|l|}{ Cardiometabolic } \\
\hline \multicolumn{5}{|l|}{ Clotting disorders } \\
\hline \multirow[t]{2}{*}{ Hepatic Factor XI } & lonis-Bayer & IONIS-FXIRx & ASO & Phase 2 [24] \\
\hline & lonis-Bayer & IONIS-FXI-LRx & ASO & Phase 1 \\
\hline \multicolumn{5}{|l|}{ Dyslipidemias } \\
\hline Hepatic PCSK9 & $\begin{array}{c}\text { Alnylam- The } \\
\text { Medicines } \\
\text { Company }\end{array}$ & $\begin{array}{l}\text { Inclisiran (ALN- } \\
\text { PCSSC) }\end{array}$ & RNAi (siRNA) & $\begin{array}{l}\text { Phase } 3 \\
\quad[16,25-28]\end{array}$ \\
\hline \multirow[t]{3}{*}{ Hepatic Apo C-III } & Arrowhead & ARO-APOC3 & RNAi (siRNA) & Phase 1 \\
\hline & $\begin{array}{c}\text { lonis-Akcea/ } \\
\text { Novartis }\end{array}$ & AKCEA-APOCIII-LRx & ASO & Phase 2 \\
\hline & lonis-Akcea & $\begin{array}{l}\text { Waylivra } \\
\text { (Volanesorsen) }\end{array}$ & ASO & $\begin{array}{l}\text { Phase } 3 \\
\text { [29-35] }\end{array}$ \\
\hline \multirow[t]{2}{*}{ Hepatic ANGPTL3 } & Arrowhead & ARO-ANG3 & RNAi (siRNA) & Phase 1 \\
\hline & lonis-Akcea & AKCEA-ANGPTL3-LRx & ASO & Phase 2 \\
\hline \multirow[t]{3}{*}{ Hepatic Apo A } & Arrowhead-Amgen & AMG 890 & RNAi (siRNA) & Phase 1 \\
\hline & $\begin{array}{l}\text { lonis-Akcea/ } \\
\text { Novartis }\end{array}$ & AKCEA-APO(a)-LRx & ASO & Phase [36] \\
\hline & Silence Therapeutics & SLN360 & & $\begin{array}{l}\text { IND/CTA due } \\
\text { in } 2020\end{array}$ \\
\hline
\end{tabular}


Table 1 (Continued)

\begin{tabular}{|c|c|c|c|c|}
\hline Disease target & $\begin{array}{l}\text { Pharmaceutical } \\
\text { company }\end{array}$ & $\begin{array}{l}\text { Drug (alternate } \\
\text { name) }\end{array}$ & $\begin{array}{l}\text { Therapeutic } \\
\text { approach }\end{array}$ & $\begin{array}{l}\text { Stage of } \\
\text { development }\end{array}$ \\
\hline Undisclosed target & lonis-AstraZeneca & $\begin{array}{c}\text { AZD8233 (IONIS- } \\
\text { AZ4-2.5LRx) }\end{array}$ & ASO & Phase 1 \\
\hline \multicolumn{5}{|l|}{ Hypertension } \\
\hline \multirow[t]{2}{*}{ Hepatic AGT } & Alnylam & ALN-AGT & RNAi (siRNA) & Phase 1 \\
\hline & lonis & IONIS-AGT-LRx & ASO & Phase 2 \\
\hline \multicolumn{5}{|l|}{ Ischemia } \\
\hline $\operatorname{miR}-92 a$ & miRagen-Servier & MRG-1 10 & $\begin{array}{l}\text { RNAi } \\
\text { (miRNA) }\end{array}$ & Phase 1 \\
\hline \multicolumn{5}{|l|}{ Type 2 diabetes } \\
\hline Hepatic glucagon receptors & lonis & IONIS-GCGR(Rx) & ASO & Phase 2 [37] \\
\hline \multicolumn{5}{|l|}{ Endocrine disorders } \\
\hline \multicolumn{5}{|l|}{ Acromegaly } \\
\hline Growth hormone receptor & lonis & IONIS-GHR-LRx & ASO & Phase 2 \\
\hline \multicolumn{5}{|l|}{ Fibrosis } \\
\hline \multicolumn{5}{|l|}{ Hypertrophic scar reduction } \\
\hline TGF- $\beta 1$ and COX2 & Sirnaomics & STP705L & RNAi (siRNA) & Phase 2 \\
\hline \multicolumn{5}{|l|}{ Cutaneous fibrosis } \\
\hline miR-29 & miRagen & Remlarsen (MRG-201) & $\begin{array}{l}\text { RNAi } \\
\quad \text { (miRNA) }\end{array}$ & Phase 2 \\
\hline
\end{tabular}

\section{Genetic}

\section{$\beta$-Thalassemia \\ Hepatic TMPRSS6}

Complement-mediated diseases

Hepatic C5

Cystic fibrosis

Pulmonary $\alpha \mathrm{ENaC}$

Hemophilia and are bleeding disorders

Hepatic antithrombin

Hereditary angioedema

Prekallikrein

Primary hyperoxaluria

Hepatic glycolate oxidase type $1 \quad$ Alnylam

Dicerna

Transthyretin-mediated (ATTR) amyloidosis

Hepatic transthyretin

\section{Alnylam}

lonis-Akcea

\footnotetext{
Kidney diseases

Delayed graft function

Pro-apoptotic gene p53

AKI following cardiac surgery

Pro-apoptotic gene p53
}

Quark
QRK306 (QPI-1002)

IONIS-TMPRSS6-LRx

SLN124

Cemdisiran (ALN-

CC5)

Cemdisiran/

Pozelimab Combo

IONIS-FB-LRx

ARO-ENaC

IONIS-ENAC-2.5Rx

Fitusiran (ALN-AT3)

IONIS-PKK-LRx

Lumasiran (ALN-GO1)

DCR-PHXC

Vutrisiran (ALN-

TTRsc02)

AKCEA-TTR-LRx

QRK209 (QPI-1002)
ASO

RNAi (siRNA)

Phase 2

CTA approved

RNAi (siRNA) Phase 2

RNAi (siRNA) Phase 1/2

ASO

Phase 2

RNAi (siRNA) Pre-IND

ASO

Phase 2

RNAi (siRNA) Phase 3

ASO

Phase 2

RNAi (siRNA) Phase 3

RNAi (siRNA) Phase 1

RNAi (siRNA) Phase 3

ASO

Phase 1

Quark

Phase 2 
Table 1 (Continued)

\begin{tabular}{|c|c|c|c|c|}
\hline Disease target & $\begin{array}{l}\text { Pharmaceutical } \\
\text { company }\end{array}$ & $\begin{array}{l}\text { Drug (alternate } \\
\text { name) }\end{array}$ & $\begin{array}{l}\text { Therapeutic } \\
\text { approach }\end{array}$ & $\begin{array}{l}\text { Stage of } \\
\text { development }\end{array}$ \\
\hline \multicolumn{5}{|l|}{ LIVER DISORDERS } \\
\hline \multicolumn{5}{|l|}{ AATD-associated liver disease } \\
\hline \multirow[t]{3}{*}{ Hepatic mutant AAT } & Arrowhead & ARO-AAT & RNAi (siRNA) & Phase 2 \\
\hline & Alnylam & ALN-AAT02 & RNAi (siRNA) & Phase $1 / 2$ \\
\hline & Dicerna & DCR-A IAT & RNAi (siRNA) & Pre-IND \\
\hline \multicolumn{5}{|l|}{ Acute hepatic porphyria } \\
\hline Hepatic ALAS1 & Alnylam & Givosiran (ALN-AS1) & RNAi (siRNA) & Phase 3 [38] \\
\hline \multicolumn{5}{|l|}{ ARLD/NAFLD } \\
\hline HSD $17 B 13$ & Arrowhead & ARO-HSD & RNAi (siRNA) & Pre-IND \\
\hline \multicolumn{5}{|l|}{$\mathrm{HBV}$} \\
\hline \multirow[t]{6}{*}{ HBV gene products } & Arrowhead- Janssen & JNJ-3989 (ARO-HBV) & RNAi (siRNA) & Phase 2 \\
\hline & Arbutus & AB-729 & RNAi (siRNA) & Phase 1 \\
\hline & Alnylam & $\begin{array}{l}\text { VIR-2218 (ALN- } \\
\text { HBVO2) }\end{array}$ & RNAi (siRNA) & Phase $1 / 2$ \\
\hline & lonis-GSK & IONIS-HBV-LRx & ASO & Phase 2 \\
\hline & lonis-GSK & IONIS-HBVRx & ASO & Phase 2 \\
\hline & Dicerna & DCR-HBVS & RNAi (siRNA) & Phase 1 \\
\hline \multicolumn{5}{|l|}{ Neurological disorders } \\
\hline \multicolumn{5}{|l|}{ Huntington's disease } \\
\hline Huntingtin protein & lonis-Roche & IONIS-HTTRx & ASO & Phase 3 [39] \\
\hline \multicolumn{5}{|l|}{ Amyotrophic lateral sclerosis } \\
\hline SODI & lonis-Biogen & Tofersen (BIIB067) & ASO & Phase $3[40]$ \\
\hline Mutated CORF72 gene & lonis-Biogen & IONIS-C9Rx (BIIB078) & ASO & Phase 2 \\
\hline \multicolumn{5}{|c|}{$\begin{array}{l}\text { Alzheimer's disease and } \\
\text { frontotemporal degeneration }\end{array}$} \\
\hline MAPT & lonis-Biogen & $\begin{array}{l}\text { IONIS-MAPTRx } \\
\text { (BIIB080) }\end{array}$ & ASO & Phase 2 \\
\hline \multicolumn{5}{|l|}{ Centronuclear myopathy } \\
\hline Dynamin 2 protein & lonis-Dynacure & IONIS-DNM2-2.5Rx & ASO & Phase 1 \\
\hline \multicolumn{5}{|l|}{ Ocular disorders } \\
\hline \multicolumn{5}{|l|}{ Dry eye syndrome } \\
\hline TRPV 1 & Sylentis & Tivanisiran (SYL1001) & RNAi (siRNA) & Phase 3 \\
\hline \multicolumn{5}{|l|}{ Glaucoma } \\
\hline$\beta$-adrenergic receptor 2 & Sylentis & $\begin{array}{l}\text { Bamosiran } \\
\text { (SLYO40012) }\end{array}$ & RNAi (siRNA) & $\begin{array}{l}\text { Phase } 2 \\
\quad[41,42]\end{array}$ \\
\hline
\end{tabular}

$\alpha \mathrm{ENaC}$, epithelial sodium Channel alpha subunit; AAT, Alpha-1 antitrypsin; AATD, Alpha-1 antitrypsin deficiency; AGT, angiotensinogen; AKI, acute kidney injury; ALAS1, aminolevulinic acid synthase 1; ANGPTL3, angiopoietin-like protein 3; Apo A, apolipoprotein A; Apo C-III, apolipoprotein C-III; ARLD/NAFLD, Alcohol-related liver disease and nonalcoholic fatty liver disease; ASO, antisense oligonucleotide; C5, complement component 5; CORF72, chromosome 9 open reading frame 72 ; CTA, clinical trial authorization; HBV, hepatitis B virus; HIF-2 $\alpha$, hypoxia inducible factor $2 \alpha$; HSD 17B 13, 17 $\beta$-Hydroxysteroid dehydrogenase type 13; IND, United States Food and Drug Administration investigational new drug application; MAPT, microtubule-associated protein tau; miRNA, microRNA; PCSK9, proprotein convertase subtilisin kexin type 9; RNAi, RNA interference; siRNA, small interfering RNA; SOD1, superoxide dismutase 1; STAT3, signal transducer and activator of transcription factor 3; TMPRSS6, Transmembrane protease serine 6; TRPV1, Transient receptor potential vanilloid-1.

improve the delivery and safety of siRNA is bioconjugation, the covalent connection of siRNAs with biogenic molecules, such as lipophilic molecules, antibodies, aptamers, ligands, peptides, or polymers, which also cause less immunoreaction $[12,13]$. The development of trivalent $\mathrm{N}$-acetylgalactosamine (GalNAc)-siRNA conjugates for targeted delivery of siRNA to the liver is the most successful. GalNAc binds to the asialoglycoprotein receptor that is highly expressed on hepatocytes resulting in rapid endocytosis [14]. The GalNAc approach has also been employed to enhance ASO delivery to hepatocytes by 10-fold versus free ASOs in preclinical models, resulting in a significant dose reduction [15]. In a phase 2 clinical trial, hepatocyte-directed GalNAcsiRNA molecules have been shown to yield incredible 
results with stable suppression of proprotein convertase subtilisin/kexin type 9 (PCSK9) achieved for at least 6 months [16]. RNA-based therapies will not only fundamentally change the way we treat diseases, but may also provide treatments for diseases with unmet clinical need. This has led to RNA-based therapeutics becoming one of the most rapidly advancing fields in drug discovery.

\section{CURRENT APPLICATION OF RNA-BASED THERAPIES}

To date, four ASOs and one siRNA-based therapy have been approved for clinical use. Fomivirsen, an intravitreally injected inhibition ASO indicated for the treatment of ocular cytomegalovirus retinitis (CMV) in acquired immunodeficiency syndrome (AIDS) individuals, was the first ever RNA-based therapy to be approved by the US Food and Drug Administration (FDA) in 1998 and by the European Medical Association (EMA) in 1999 [17]. However, in the early 2000s, fomivirsen was withdrawn from the market as the introduction of highly active antiretroviral therapy (HAART) dramatically reduced the number of cases of CMV. A second inhibition ASO, mipomersen, which is injected subcutaneously and targets mRNA encoding apolipoprotein B for the treatment of homozygous familial hypercholesterolemia, was approved for clinical use in 2014 [18]. Two splice-modulating ASOs, nusinersen for the treatment of spinal muscular atrophy (2017) $[19,20]$ and eteplirsen for the treatment of Duchenne muscular dystrophy (2016) [21], are also approved for clinical use. In 2018, patisiran, an siRNA encapsulated in a lipid nanoparticle for delivery to hepatocytes, became the first globally (FDA and EMA)-approved RNAi therapeutic. Patisiran is indicated for the treatment of hereditary transthyretin-mediated amyloidosis (hATTR) in adults [22]. In addition, more than 50 RNA-based therapies for diseases as varied as cancer, neurodegenerative disease and cardiovascular disease are currently in clinical development with several investigational new drug and clinical trial applications expected to be filed within the next 2 years (Table 1). Interestingly, a number of ASO and RNAi therapies are being developed for the same disease targets (Table 1), including hepatic AGT for the treatment of hypertension, which are in phase 2 and phase 1 , respectively.

\section{ANGIOTENSINOGEN RNA-BASED THERAPY IN HYPERTENSION}

As AGT is the sole precursor of the potent vasoconstrictor Ang II, it is a promising target for gene silencing. At present, inhibition of the renin-Angaldosterone system (RAAS) is the mainstay for the treatment of hypertension, heart failure and chronic kidney disease $[43,44]$. Yet, the long-term management of these conditions remains complicated by RAAS escape phenomena, during which a counterregulatory rise in plasma renin often restores Ang II to its original pretreatment values [45-47]. As a consequence, blood pressure control commonly requires multiple antihypertensive drugs, but is impeded by a decline in therapy adherence with every drug added to the treatment regimen $[48,49]$.

The clinical need for an antihypertensive therapeutic, which prevents Ang reactivation has long been recognized and may be achieved by blocking the RAAS at the AGT level. As opposed to classical RAAS inhibitors, a compensatory rise in renin could even potentiate blood pressure lowering as it would facilitate depletion of the remaining AGT (Fig. 2a). This can be explained as follows. Normally, AGT is present at levels that exceed Ang levels by many orders of magnitude. Under such conditions, blocking renin, Ang I-converting enzyme (ACE) or the Ang receptor results in a renin rise, which either (partially) restores Ang II, or increases Ang II above its original level (in order to overcome blockade of its receptor). This is easily achievable, simply because there is ample AGT around to allow this. Even a 100-fold rise in Ang I generation (sufficient to overcome 99\% renin inhibition!) is feasible. Yet, if the renin rises are substantial, it is possible that at a certain moment, AGT levels start to decrease. During AGT suppression by RNA-based therapy, AGT levels are down already, and now a rise in renin might be sufficient to result in complete AGT depletion, particularly when simultaneously introducing other types of RAAS blockade, which induce further rises in renin. Restoration of Ang II levels is then no longer possible.

Although initial ASO constructs targeting Agt lowered circulating AGT, Ang II and blood pressure in parallel in spontaneously hypertensive rats, these methods lacked potency and specificity: AGT levels were halved at most, and the effects were short-lived ( $<1$ week) [51-53]. Similar problems also impeded the use of Agt-directed siRNA. The introduction of lipid nanoparticle delivery enhanced AGT downregulation, although stable suppression still necessitated weekly intravenous dosing [54]. Additionally, treatment with lipid delivery vehicles causes an inflammatory response, and must therefore, be given with histamine receptor antagonists, nonsteroidal anti-inflammatory drugs and relatively high doses of glucocorticoids [55].

Conjugating GalNAc to ASO and RNAi molecules enabled general therapeutic application. Subsequent selective accumulation in the liver permitted minimal 


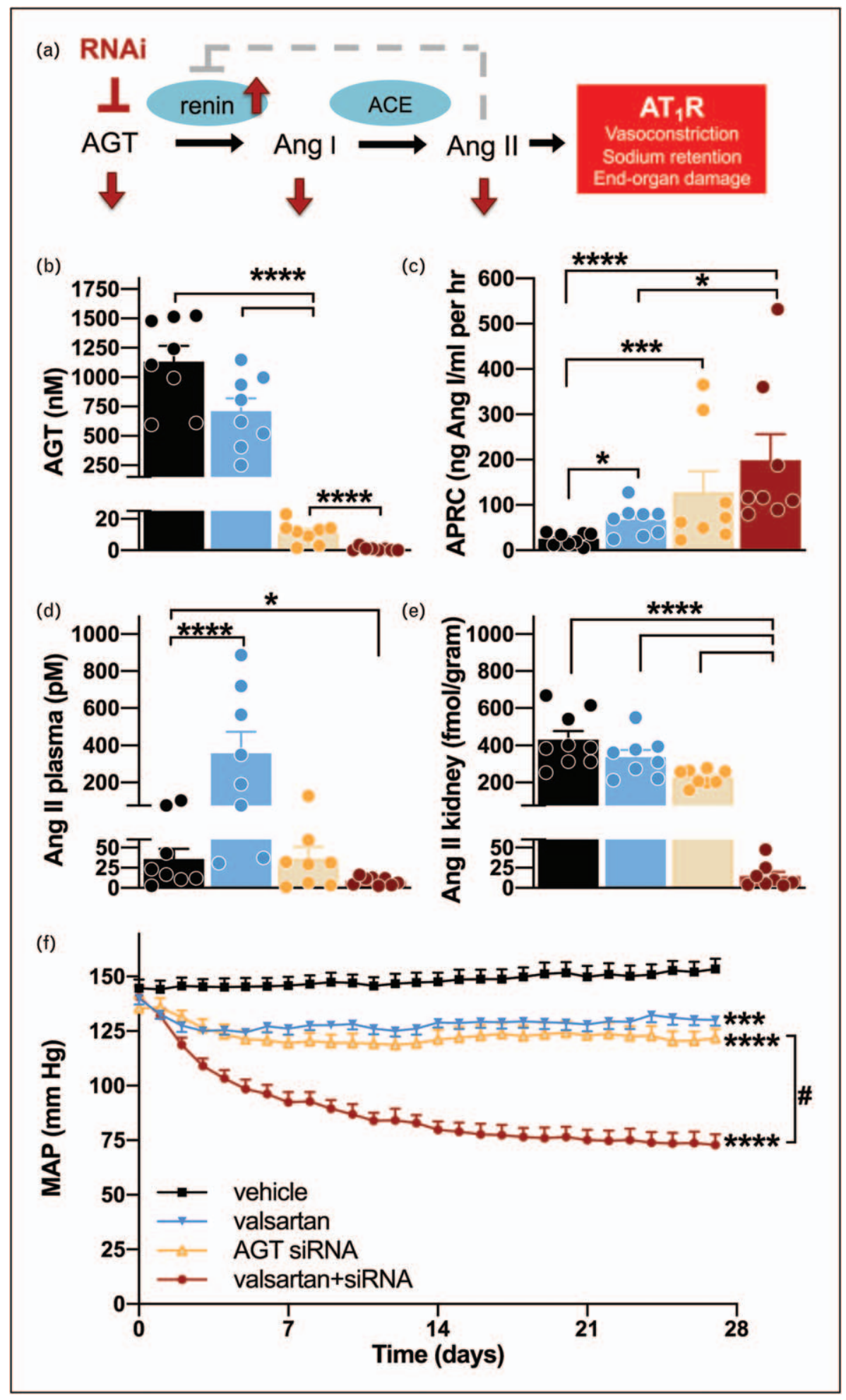

FIGURE 2. Near elimination of angiotensinogen is required for lowering of Ang II. (a) Inhibition of the renin-angiotensinaldosterone system (RAAS) at the angiotensinogen (AGT) level may prevent RAAS reactivation, because a rise in plasma renin levels, because of a lack of negative feedback exerted by angiotensin (Ang) II on renin production, could now facilitate depletion of remaining AGT. (b) Plasma AGT, (c) active plasma renin concentration (APRC), (d) plasma Ang II, (e) renal Ang II and (f) mean arterial pressure (MAP) of spontaneously hypertensive rats after 4 weeks of vehicle, valsartan, AGT siRNA or valsartan with siRNA treatment (all groups $n=8$ ). Data, modified from Uij et al. [50"-], are represented as means \pm SEM and analyzed using two-way ANOVA and posthoc Bonferroni (MAP) or transformed to natural logarithms before analysis by one-way ANOVA and posthoc Bonferroni (RAS parameters). ${ }^{*} P<0.05,{ }^{* * *} P \leq 0.001,{ }^{* * * *} P \leq 0.0001$ vs. vehicle or otherwise indicated; ${ }^{\#} P \leq 0.0001$. 
invasive delivery via subcutaneous injection and optimization of AGT downregulation, as circulating AGT levels are determined by hepatic synthesis [56,57"]. Indeed, by improving liver activity eight-fold, GalNAc-conjugation potentiated AGT inhibition of ASOs to maximally $88 \%$ in a dose-dependent manner [58]. Interestingly, a reduction of at least $75 \%$ was required to simultaneously lower blood pressure, which then remained low for a period of 7-10 days after a single dose. This relates to the above-mentioned upregulation of renin. In fact, in a study employing GalNAcsiRNA to target $A g t$ [50"'], even when AGT was decreased by $97.9 \%$, circulating Ang II remained intact because of renin upregulation. Only a $99.8 \%$ reduction, achieved by combining siRNA with valsartan (which further upregulates renin, thereby additionally lowering AGT), was required to deplete both systemic and renal Ang II (Fig. 2b-e). The latter observation implies that renal Ang generation relies on AGT of hepatic origin. Consequently, AGT siRNA monotherapy provided similar antihypertensive and cardioprotective efficacy as conventional RAAS inhibition, whereas near elimination of AGT by addition of valsartan yielded a synergistic reduction in blood pressure and cardiac hypertrophy, without adversely affecting renal function (Fig. 2f). Unique to GalNAc-siRNA is its long-lasting effectiveness [22], as opposed to an ASO liver half-life of 2-4 weeks [58]. These findings suggest that, in clinical practice, AGT inhibition mediated by RNAi may not only prevent RAAS reactivation but also improve cardiovascular outcome and therapy adherence because of a sustained and stable single-dose efficacy lasting weeks to months [50"'].

\section{ANGIOTENSINOGEN RNA-BASED THERAPY IN OTHER DISEASES}

Although the potential for clinical translation is currently being investigated in patients, preclinical studies now focus on safety and efficacy in models of chronic kidney disease and heart failure. However, the application of AGT inhibition is not limited to hypertension and related end-organ damage. Polycystic kidney disease (PKD) appears an especially promising target, with multiple recent studies showing beneficial effects in animal models of this disease $\left[59,60,61^{\prime \prime}\right.$. PKD is caused by mutations in the $P k d 1$ and $P k d 2$ genes. In affected patients, these mutations lead to early onset hypertension, the progressive growth of renal cysts and kidney failure. Conventional treatment with ACE inhibitors or Ang receptor blockers (ARBs) effectively lowers blood pressure in PKD, but whether this treatment also reduces the decline in kidney function is unclear $[62,63]$. Experimentally, PKD may be studied in mice with targeted knockout of the $P k d 1$ or $P k d 2$ gene, with both models developing progressive intrarenal cysts and kidney failure [64,65]. In $P k d 2$ knockout mice, treatment with AGT ASO resulted in a reduction in kidney size, lower total cyst volume and improved kidney function compared with control treatment (scrambled ASO) [59]. Pkd1 knockout mice displayed similar reductions in kidney size and cyst volume after treatment with AGT ASO [60,61"]. Interestingly, in the latter model, the effects of AGT ASO on cyst growth were independent of changes in SBP. In contrast, ACE inhibition with lisinopril significantly lowered SBP, but did not reduce kidney size to a similar degree as AGT ASO and had no effect on cyst volume [60].

Experimental evidence indicates that AGT ASO may also be effective in the treatment of atherosclerosis. Increased activity of the RAAS promotes atherosclerosis through activation of $\mathrm{AT}_{1 \mathrm{~A}}$ receptors [66-68]. Treatment of spontaneously hypertensive rats on a diet enriched with high fat and vitamin D3 (i.e. to induce atherosclerosis) with Gal-PEG-Et (GPE) nanoparticles carrying short hairpin RNA (shRNA) that specifically targets Agt in the liver decreased SBP and attenuated the development of atherosclerotic lesions compared with control animals [69]. Similar effects were observed in another animal model for atherosclerosis and obesity, that is, LDL receptor knockout mice fed with a saturated fat-enriched diet [70]. In these mice, treatment with AGT ASO resulted in decreased blood pressure, less atherosclerosis, and diminished body weight gain. Correspondingly, previous clinical studies also showed beneficial effects of ACE inhibitors and ARBs on the progression of atherosclerotic plaques [71,72]. However, whether AGT ASO is effective in the treatment of atherosclerosis in patients has yet to be investigated.

Finally, AGT ASO treatment has been used as a therapeutic strategy against experimental pulmonary fibrosis. Currently, treatment options for idiopathic pulmonary fibrosis are limited, leaving most patients with a poor prognosis - the median survival rate is $2-5$ years. Several studies in animals (i.e., mice and rats) with bleomycin-induced lung injury showed that Ang II contributes to pulmonary fibrosis, whereas treatment with renin inhibitors, ACE inhibitors, and ARBs attenuates disease progression [73-77]. In this same animal model, intratracheal administration of AGT ASO was found to decrease pulmonary AGT concentrations and lung fibrosis [78], but no comparison was made to conventional RAAS inhibitors.

\section{CONCLUSION}

Targeting AGT with RNA-based therapeutics is a promising new tool to treat hypertension and diseases beyond, including heart failure, atherosclerosis, 
diabetic nephropathy, polycystic kidney disease and pulmonary fibrosis. The long-lasting effects of RNAbased therapeutics are particularly exciting, and if translated to a clinical application of at most a few administrations per year, may help to eliminate nonadherence. To what degree the effects of such therapeutics are fully identical to those of other RAAS blockers (given the fact that they eliminate all Ang metabolites) remains to be determined. It will also be important to determine to what level AGT should be suppressed to induce meaningful effects, and whether there is a role for AGT synthesized outside the liver [79]. Synergy in combination with other RAAS blockers needs to be evaluated, as well as the potential harmful effects of suppressing AGT in the context of common comorbidities, such as heart failure and chronic kidney disease, where AGT might already be suppressed.

\section{Acknowledgements}

None.

\section{Financial support and sponsorship}

K.M.M.C. was supported by a National Health and Medical Research Council of Australia CJ Martin Fellowship (1112125). L.R. was supported by a National Natural Science Foundation of China grant (no. 81900668).

\section{Conflicts of interest}

A.H.J.D. received a grant from Alnylam Pharmaceuticals.

\section{REFERENCES AND RECOMMENDED READING}

Papers of particular interest, published within the annual period of review, have been highlighted as:

- of special interest

-1. of outstanding interest

1. Balcarek J, Sevá Pessôa B, Bryson C, et al. Multiple ascending dose study with the new renin inhibitor VTP-27999: nephrocentric consequences of too much renin inhibition. Hypertension 2014; 63:942-950.

2. Setten RL, Rossi JJ, Han SP. The current state and future directions of RNAi-

n based therapeutics. Nat Rev Drug Discov 2019; 18:421-446.

Excellent review on the design and development of RNAi drugs.

3. Levin AA. Treating disease at the RNA level with oligonucleotides. N Engl J

- Med 2019; 380:57-70.

Overview of therapeutic oligonucleotides at this moment.

4. Liang $X H$, Sun $H$, Nichols JG, Crooke ST. RNase H1-dependent antisense oligonucleotides are robustly active in directing RNA cleavage in both the cytoplasm and the nucleus. Mol Ther 2017; 25:2075-2092

5. Wang $\mathrm{S}$, Allen N, Vickers TA, et al. Cellular uptake mediated by epidermal growth factor receptor facilitates the intracellular activity of phosphorothioatemodified antisense oligonucleotides. Nucleic Acids Res 2018; 46: 3579-3594.

6. Wang S, Allen N, Liang XH, Crooke ST. Membrane destabilization induced by lipid species increases activity of phosphorothioate-antisense oligonucleotides. Mol Ther Nucleic Acids 2018; 13:686-698.

7. Li C, Zamore PD. RNA interference and small RNA analysis. Cold Spring Harb Protoc 2019; 2019:dbto097436.

8. Daugaard I, Hansen TB. Biogenesis and function of ago-associated RNAs. Trends Genet 2017; 33:208-219.
9. Bertrand JR, Pottier M, Vekris A, et al. Comparison of antisense oligonucleotides and siRNAs in cell culture and in vivo. Biochem Biophys Res Commun 2002; 296:1000-1004.

10. Osborn MF, Khvorova A. Improving siRNA delivery in vivo through lipid conjugation. Nucleic Acid Ther 2018; 28:128-136.

11. Yalcin E, Kara G, Celik E, et al. Preparation and characterization of novel albumin-sericin nanoparticles as siRNA delivery vehicle for laryngeal cancer treatment. Prep Biochem Biotechnol 2019; 49:659-670.

12. Chernikov IV, Vlassov VV, Chernolovskaya EL. Current development of siRNA bioconjugates: from research to the clinic. Front Pharmacol 2019; 10:444.

13. $\mathrm{Qu} X, \mathrm{Hu} Y$, Wang $\mathrm{H}$, et al. Biomimetic dextran-peptide vectors for efficient and safe siRNA delivery. ACS Applied Bio Materials 2019; 2:1456-1463.

14. Springer AD, Dowdy SF. GalNAc-siRNA conjugates: leading the way for delivery of RNAi therapeutics. Nucleic Acid Ther 2018; 28:109-118.

15. Prakash TP, Graham MJ, Yu J, et al. Targeted delivery of antisense oligonucleotides to hepatocytes using triantennary $\mathrm{N}$-acetyl galactosamine improves potency 10-fold in mice. Nucleic Acids Res 2014; 42:8796-8807.

16. Ray KK, Landmesser U, Leiter LA, et al. Inclisiran in patients at high cardiovascular risk with elevated LDL cholesterol. N Engl J Med 2017; 376:1430-1440.

17. Roehr B. Fomivirsen approved for CMV retinitis. J Int Assoc Physicians AIDS Care 1998; 4:14-16.

18. Wong E, Goldberg T. Mipomersen (kynamro): a novel antisense oligonucleotide inhibitor for the management of homozygous familial hypercholesterolemia. P t 2014; 39:119-122.

19. Stein CA, Castanotto D. FDA-approved oligonucleotide therapies in 2017. Mol Ther 2017; 25:1069-1075.

20. Aartsma-Rus A. FDA approval of nusinersen for spinal muscular atrophy makes 2016 the year of splice modulating oligonucleotides. Nucleic Acid Ther 2017; 27:67-69.

21. Aartsma-Rus $A$, Krieg AM. FDA approves eteplirsen for duchenne muscular dystrophy: the next chapter in the eteplirsen saga. Nucleic Acid Ther 2017; 27:1-3.

22. Hoy SM. Patisiran: first global approval. Drugs $2018 ; 78: 1625-1631$.

23. Golan T, Khvalevsky EZ, Hubert $A$, et al. RNAi therapy targeting KRAS in combination with chemotherapy for locally advanced pancreatic cancer patients. Oncotarget 2015; 6:24560-24570.

24. Buller HR, Bethune $C$, Bhanot $S$, et al. Factor $X I$ antisense oligonucleotide for prevention of venous thrombosis. N Engl J Med 2015; 372:232-240.

25. Fitzgerald $\mathrm{K}$, White $S$, Borodovsky $A$, et al. A highly durable RNAi therapeutic inhibitor of PCSK9. N Engl J Med 2017; 376:41-51.

26. Ray KK, Stoekenbroek RM, Kallend D, et al. Effect of 1 or 2 doses of inclisiran on low-density lipoprotein cholesterol levels: one-year follow-up of the ORION-1 Randomized Clinical Trial. JAMA Cardiol 2019; In press.

27. Leiter LA, Teoh $\mathrm{H}$, Kallend $\mathrm{D}$, et al. Inclisiran lowers LDL-C and PCSK9 irrespective of diabetes status: the ORION-1 Randomized Clinical Trial. Diabetes Care 2019; 42:173-176.

28. Ray KK, Stoekenbroek RM, Kallend D, et al. Effect of an siRNA therapeutic targeting PCSK9 on atherogenic lipoproteins. Circulation 2018; 138:1304-1316.

29. Witztum JL, Gaudet D, Freedman SD, et al. Volanesorsen and triglyceride levels in familial chylomicronemia syndrome. N Engl J Med 2019; $381: 531-542$

30. Hegele RA, Berberich AJ, Ban MR, et al. Clinical and biochemical features of different molecular etiologies of familial chylomicronemia. J Clin Lipidol 2018; 12:920.e4-927.e4.

31. Digenio A, Dunbar RL, Alexander VJ, et al. Antisense-mediated lowering of plasma apolipoprotein C-III by volanesorsen improves dyslipidemia and insulin sensitivity in type 2 diabetes. Diabetes Care 2016; 39:1408-1415.

32. Jorgensen $A B$, Frikke-Schmidt R, Nordestgaard BG, et al. Loss-of-function mutations in APOC3 and risk of ischemic vascular disease. N Engl J Med 2014; 371:32-41.

33. Graham MJ, Lee RG, Bell TA 3rd, et al. Antisense oligonucleotide inhibition of apolipoprotein C-III reduces plasma triglycerides in rodents, nonhuman primates, and humans. Circ Res 2013; 112:1479-1490.

34. Gaudet D, Alexander VJ, Baker BF, et al. Antisense inhibition of apolipoprotein C-III in patients with hypertriglyceridemia. N Engl J Med 2015; 373:438-447.

35. Gaudet D, Brisson D, Tremblay $K$, et al. Targeting APOC3 in the familial chylomicronemia syndrome. N Engl J Med 2014; 371:2200-2206.

36. Viney NJ, van Capelleveen JC, Geary RS, et al. Antisense oligonucleotides targeting apolipoprotein(a) in people with raised lipoprotein(a): two randomised, double-blind, placebo-controlled, dose-ranging trials. Lancet 2016; 388:2239-2253.

37. Morgan ES, Tai LJ, Pham NC, et al. Antisense inhibition of glucagon receptor by IONIS-GCGRRx improves type 2 diabetes without increase in hepatic glycogen content in patients with type 2 diabetes on stable metformin therapy. Diabetes Care 2019; 42:585-593.

38. Sardh $E$, Harper $P$, Balwani $M$, et al. Phase 1 trial of an rna interference therapy for acute intermittent porphyria. N Engl J Med 2019; 380:549-558.

39. Tabrizi SJ, Leavitt BR, Landwehrmeyer GB, et al., Phase 1-2a IONIS-HTTRx Study Site Teams. Targeting Huntingtin expression in patients with Huntington's disease. N Engl J Med 2019; 380:2307-2316. 
40. Miller TM, Pestronk A, David W, et al. An antisense oligonucleotide against SOD1 delivered intrathecally for patients with SOD1 familial amyotrophic lateral sclerosis: a phase 1, randomised, first-in-man study. Lancet Neurol 2013; 12:435-442.

41. Martinez T, Gonzalez MV, Roehl I, et al. In vitro and in vivo efficacy of SYL040012, a novel siRNA compound for treatment of glaucoma. Mol Ther $2014 ; 22: 81-91$.

42. Moreno-Montanes J, Sadaba B, Ruz V, et al. Phase I clinical trial of SYL040012, a small interfering RNA targeting beta-adrenergic receptor 2, for lowering intraocular pressure. Mol Ther 2014; 22:226-232.

43. Williams B, Mancia G, Spiering W, et al. UK-TREND Eczema Network. 2018 ESC/ESH Guidelines for the management of arterial hypertension. Eur Heart J 2018; 39:3021-3104

44. Arendse LB, Danser AHJ, Poglitsch M, et al. Novel therapeutic approaches targeting the renin-angiotensin system and associated peptides in hypertension and heart failure. Pharmacol Rev 2019; 71:539-570.

45. van den Meiracker AH, Admiraal PJ, Janssen JA, et al. Hemodynamic and biochemical effects of the AT1 receptor antagonist irbesartan in hypertension. Hypertension 1995; 25:22-29.

46. Mooser V, Nussberger J, Juillerat $L$, et al. Reactive hyperreninemia is a major determinant of plasma angiotensin II during ACE inhibition. J Cardiovasc Pharmacol 1990; 15:276-282.

47. Sealey JE, Laragh JH. Aliskiren, the first renin inhibitor for treating hypertension: reactive renin secretion may limit its effectiveness. Am J Hypertens 2007; 20:587-597.

48. Gupta $P$, Patel $P$, Strauch $B$, et al. Risk factors for nonadherence to antihypertensive treatment. Hypertension 2017; 69:1113-1120.

49. Cushman WC, Ford CE, Cutler JA, et al., ALLHAT Collaborative Research Group. Success and predictors of blood pressure control in diverse North American settings: the antihypertensive and lipid-lowering treatment to prevent heart attack trial (ALLHAT). J Clin Hypertens (Greenwich) 2002; 4:393-404.

50. Uij E, Mirabito Colafella KM, Sun Y, et al. Strong and sustained antihyper-

- tensive effect of small interfering RNA targeting liver angiotensinogen. Hypertension 2019; 73:1249-1257.

Extensive biochemical evaluation of angiotensinogen siRNA in hypertensive rats.

51. Tomita N, Morishita R, Higaki J, et al. Transient decrease in high blood pressure by in vivo transfer of antisense oligodeoxynucleotides against rat angiotensinogen. Hypertension 1995; 26:131-136.

52. Wielbo D, Simon A, Phillips MI, et al. Inhibition of hypertension by peripheral administration of antisense oligodeoxynucleotides. Hypertension 1996; 28:147-151.

53. Makino N, Sugano M, Ohtsuka S, Sawada S, et al. Intravenous injection with antisense oligodeoxynucleotides against angiotensinogen decreases blood pressure in spontaneously hypertensive rats. Hypertension 1998; $31: 1166-1170$.

54. Olearczyk J, Gao S, Eybye M, et al. Targeting of hepatic angiotensinogen using chemically modified siRNAs results in significant and sustained blood pressure lowering in a rat model of hypertension. Hypertens Res 2014; 37:405-412.

55. Crooke ST, Witztum JL, Bennett CF, et al. RNA-targeted therapeutics. Cell Metab 2018; 27:714-739.

56. Matsusaka T, Niimura F, Shimizu A, et al. Liver angiotensinogen is the primary source of renal angiotensin II. J Am Soc Nephrol 2012; 23:1181-1189.

57. $\mathrm{Ye} F, \mathrm{Wang} \mathrm{Y}, \mathrm{Wu} \mathrm{C}$, et al. Angiotensinogen and megalin interactions

- contribute to atherosclerosis-brief report. Arterioscler Thromb Vasc Biol 2019; 39:150-155.

First evaluation of angiotensinogen antisense oligonucleotide in atherosclerosis.

58. Mullick AE, Yeh ST, Graham MJ, et al. Blood pressure lowering and safety improvements with liver angiotensinogen inhibition in models of hypertension and kidney injury. Hypertension 2017; 70:566-576.

59. Ravichandran $K$, Ozkok $A$, Wang $Q$, et al. Antisense-mediated angiotensinogen inhibition slows polycystic kidney disease in mice with a targeted mutation in Pkd2. Am J Physiol Renal Physiol 2015; 308:F349-F357.
60. Saigusa $T$, Dang $Y$, Mullick $A E$, et al. Suppressing angiotensinogen synthesis attenuates kidney cyst formation in a Pkd1 mouse model. FASEB J 2016; 30:370-379.

61. Fitzgibbon WR, Dang $Y$, Bunni MA, et al. Attenuation of accelerated renal

- cystogenesis in Pkd1 mice by renin-angiotensin system blockade. Am J Physiol Renal Physiol 2018; 314:F210-F218.

Evaluation of angiotensinogen antisense oligonucleotide in a polycystic kidney disease mouse model.

62. Jafar $\mathrm{TH}$, Stark $\mathrm{PC}$, Schmid $\mathrm{CH}$, et al., ACE Inhibition in Progressive Renal Disease (AIPRD) Study Group. The effect of angiotensin-converting-enzyme inhibitors on progression of advanced polycystic kidney disease. Kidney Int 2005; 67:265-271.

63. Irazabal MV, Abebe KZ, Bae KT, et al., HALT Investigators. Prognostic enrichment design in clinical trials for autosomal dominant polycystic kidney disease: the HALT-PKD clinical trial. Nephrol Dial Transplant 2017; 32:1857-1865.

64. Piontek KB, Huso DL, Grinberg A, et al. A functional floxed allele of Pkd1 that can be conditionally inactivated in vivo. J Am Soc Nephrol 2004; 15:3035-3043.

65. Wu G, D'Agati V, Cai Y, et al. Somatic inactivation of $P k d 2$ results in polycystic kidney disease. Cell 1998; 93:177-188.

66. Cassis LA, Rateri DL, Lu H, et al. Bone marrow transplantation reveals that recipient AT1a receptors are required to initiate angiotensin II-induced atherosclerosis and aneurysms. Arterioscler Thromb Vasc Biol 2007; 27:380-386.

67. Daugherty A, Rateri DL, Lu H, et al. Hypercholesterolemia stimulates angiotensin peptide synthesis and contributes to atherosclerosis through the AT1 A receptor. Circulation 2004; 110:3849-3857.

68. Eto $\mathrm{H}$, Miyata $\mathrm{M}$, Shirasawa $\mathrm{T}$, et al. The long-term effect of angiotensin Il type 1a receptor deficiency on hypercholesterolemia-induced atherosclerosis. Hypertens Res 2008; 31:1631-1642.

69. Lu $P$, Yuan $L$, Wang $Y$, et al. Effect of GPE-AGT nanoparticle shRNA transfection system mediated RNAi on early atherosclerotic lesion. Int J Clin Exp Pathol 2012; 5:698-706.

70. Lu H, Wu C, Howatt DA, et al. Angiotensinogen exerts effects independent of angiotensin II. Arterioscler Thromb Vasc Biol 2016; 36:256-265.

71. Lonn E, Yusuf S, Dzavik V, et al. Effects of ramipril and vitamin $E$ on atherosclerosis: the study to evaluate carotid ultrasound changes in patients treated with ramipril and vitamin E (SECURE). Circulation 2001; 103:919-925.

72. Shinoda $E$, Yui $Y$, Kodama $K$, et al., Japan Multicenter Investigation for Cardiovascular Diseases-B Study Group. Quantitative coronary angiogram analysis: nifedipine retard versus angiotensin-converting enzyme inhibitors (JMIC-B side arm study). Hypertension 2005; 45:1153-1158.

73. Marshall RP, Gohlke P, Chambers RC, et al. Angiotensin II and the fibroproliferative response to acute lung injury. Am J Physiol Lung Cell Mol Physiol 2004; 286:L156-L164.

74. Otsuka M, Takahashi $H$, Shiratori $M$, et al. Reduction of bleomycin induced lung fibrosis by candesartan cilexetil, an angiotensin II type 1 receptor antagonist. Thorax 2004; 59:31-38.

75. Li X, Rayford H, Uhal BD. Essential roles for angiotensin receptor AT1a in bleomycin-induced apoptosis and lung fibrosis in mice. Am J Pathol 2003; 163:2523-2530.

76. Wang R, lbarra-Sunga $O$, Verlinski L, et al. Abrogation of bleomycin-induced epithelial apoptosis and lung fibrosis by captopril or by a caspase inhibitor. Am J Physiol Lung Cell Mol Physiol 2000; 279:L143-151.

77. Asker SA, Mazroa SA, Boshra V, et al. Hassan AM. Biochemical and histological impact of direct renin inhibition by aliskiren on myofibroblasts activation and differentiation in bleomycin induced pulmonary fibrosis in adult mice. Tissue Cell 2015; 47:373-381.

78. Li X, Zhuang J, Rayford $\mathrm{H}$, et al. Attenuation of bleomycin-induced pulmonary fibrosis by intratracheal administration of antisense oligonucleotides against angiotensinogen mRNA. Curr Pharm Des 2007; 13:1257-1268.

79. Ren L, Lu X, Danser AHJ. Revisiting the brain renin-angiotensin system-focus on novel therapies. Curr Hypertens Rep 2019; 21:28. 